\title{
3 Research Suare

\section{Constitutive WNT Signaling via CTNNB1 Stabilization in the Yolk Sac Epithelium Results in Embryonic Lethality in Mice}

\section{Mayur Bansal}

Texas A\&M University College Station

\section{Danila Cuomo}

Texas A\&M University College Station

Ephraim A. Yusi

Texas A\&M University College Station

Rachel M. Lynch

Texas A\&M University College Station

David Threadgill ( $\nabla$ dwt@tamu.edu )

Texas A\&M University College Station https://orcid.org/0000-0003-3538-1635

Research article

Keywords: yolk sac, beta-catenin, hematopoiesis, villin

Posted Date: December 17th, 2020

DOl: https://doi.org/10.21203/rs.3.rs-88503/v1

License: (9) This work is licensed under a Creative Commons Attribution 4.0 International License. Read Full License 


\section{Abstract}

\section{Background}

Beta-catenin (CTNNB1) is a key downstream effector of the WNT signaling pathway that is involved in embryonic development and tumorigenesis. To further study the role of CTNNB1 in gut tumorigenesis, mice carrying a conditional, stabilizing mutation of Ctnnb1 (Ctnnb1 ${ }^{t m} 1 \mathrm{Mmt}$ ) were crossed with mice expressing CRE recombinase under the intestinal epithelial Villin 1 promoter (Vil1-Cre).

\section{Results}

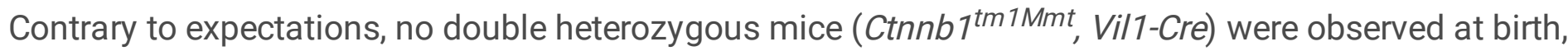
suggesting embryonic lethality. After assessment of the timing of embryonic lethality, which occurs between embryonic stage 11.5 and 13.5, the (ROSA)26Sor ${ }^{\text {tm } 1 \text { Sor }}$ reporter was crossed with Vil1-Cre and the resulting embryos and placentae were stained for beta-galactosidase activity. CRE under control of the Vil1 promoter was found to be expressed in the yolk sac epithelium, indicating potential extraembryonic defects caused by constitutive WNT signaling. Analysis of RNAseq data from yolk sacderived RNA indicated changes in inflammation-associated pathways due to the constitutive WNT signaling.

\section{Conclusions}

Embryonic lethality due to Vil1-Cre driven WNT signaling in the yolk sac epithelium causes potential defects in the transfer of yolk sac derived erythromyeloid progenitor cells and hematopoietic stem cells to the embryo, and shows the limits of using the Vil1-Cre transgenic line for analysis of adult tissues.

\section{Background}

Beta-catenin (CTNNB1) has crucial roles during both embryonic development and tumorigenesis, with two distinct functions depending on its location. At the cellular membrane, CTNNB1 is involved in the proper formation of adherens junctions between cells [1], while in the cytoplasm, CTNNB1 translocates to the nucleus and functions as a transcriptional co-activator during WNT signaling [2]. Ctnnb $1^{\text {tm } 1 M m t}$ has garnered significant clinical interest because of its involvement in tumorigenesis. It is estimated that $10 \%$ of all cancers have mutations in Ctnnb $1^{\operatorname{tm} 1 \mathrm{Mmt}}$ [3], with up to $90 \%$ of all colorectal cancers (CRC) having aberrant WNT signaling, including mutations in CTNNB1 or other members of the WNT pathway [4].

Cytoplasmic concentrations of CTNNB1 are regulated through ubiquitination by the E3 ubiquitin ligase BTRC [5]. However, before BTRC can bind CTNNB1, it is phosphorylated by the glycogen synthase kinase GSK3B [5]. The phosphorylation sites for GSK3B are encoded by exon 3 in Ctnnb ${ }^{\text {tm } 1 M m t}$ [6]. When exon 3 is deleted or the GSK3B phosphorylation site is mutated, as occurs in many cancers [7], CTNNB1 can no longer be targeted for ubiquitination and is stabilized. Similarly, upon WNT activation, the AXIN complex, including GSK3 that is responsible for the degradation of CTNNB1, is recruited to receptors on the 
intracellular side of the cell membrane, resulting in the inhibition of CTNNB1 ubiquitination [8]. Upon stabilization, CTNNB1 can enter the nucleus and function as a transcriptional co-activator [8].

To develop a model of CTNNB1-driven tumorigenesis in the intestinal tract, we crossed mice carrying Vil1Cre with mice carrying a conditional, stabilizing mutation in Ctnnb $1^{t m 1 M m t}$. Unexpectedly, the double mutants were embryonic lethal. We present data on an embryonic lethality resulting from stabilization of CTNNB1 in the yolk sac epithelium and propose that embryonic lethality is due to defects in the transfer of yolk sac derived erythromyeloid progenitor cells (EMPs) and hematopoietic stem cells (HSCs) to the embryo.

\section{Results}

Excision of exon 3 from $\mathrm{Ctnnb} 1^{\mathrm{tm} 1 \mathrm{Mmt}}$ by Vil1-Cre leads to embryonic lethality

To further understand the role CTNNB1 plays during intestinal cancer development, mice carrying a Ctnnb $1^{\text {tm 1Mmt }}$, a conditional, stabilizing mutation in Ctnnb $1^{\text {tm } 1 M m t}$, were crossed with mice carrying Vil1Cre. Mice carrying both Vil1-Cre and Ctnnb $1^{\text {tm } 1 \mathrm{Mmt}}$ alleles were not recovered at birth suggesting that they were embryonic lethal (Table 1).

Table 1

Genotypes resulting from Ctnnb $1^{\text {tm } 1 M m t}$ by Vil1-Cre crosses.

\begin{tabular}{|lllll|}
\hline $\begin{array}{l}\text { Days Post } \\
\text { Coitus }\end{array}$ & $\begin{array}{l}\text { Number of Total } \\
\text { Embryos/Mice }\end{array}$ & $\begin{array}{l}\text { Ctnnb1tm1Mmt \% } \\
\text { of Total }\end{array}$ & $\begin{array}{l}\text { Ctnnb1 } \\
\text { Vilm-Cre } \\
\text { \% of Total }\end{array}$ & $\begin{array}{l}\text { Ctnnb1 } \\
\text { Cre Viable } \\
\text { \% of Total }\end{array}$ \\
\hline E10.5 & 29 & 52 & 57 & 100 \\
\hline E11.5 & 9 & 56 & 44 & 100 \\
\hline E12.5 & 7 & 43 & 57 & 43 \\
\hline E13.5 & 52 & 54 & 46 & 17 \\
\hline PND21 & 27 & 27 & 0 & 0 \\
\hline (Weaning) & & & & \\
\hline
\end{tabular}

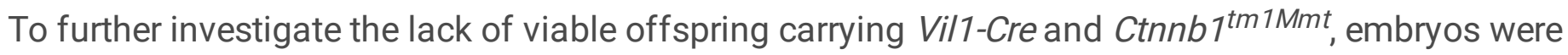
collected between E10.5 and E13.5 and visually analyzed for aberrant development and reabsorption. Double mutants showed dramatic differences in development by E12.5 (Fig. 1A), with hemorrhaging and visible size differences. By E13.5 most of the embryos were at various stages of reabsorption.

Vil1 promoter expresses CRE in the yolk sac epithelium 
In order to identify possible causes of the embryonic lethality, the mice carrying the (ROSA)26Sor ${ }^{\text {tm } 1 \text { Sor }}$ reporter were crossed with mice carrying Vil1-Cre. Embryos and extraembryonic tissues were collected and stained for beta-galactosidase activity, which showed beta-galactosidase activity in the yolk sac epithelium (Fig. 1B). No expression was observed in embryos. This result is consistent with lethality at E12.5 being caused by abnormal function of extraembryonic tissues. Consistent with these results, previous studies have shown that CTNNB1 signaling in the yolk sac epithelium plays an essential role in the activation and maintenance of embryonic hematopoiesis $[9,10]$.

\section{Inflammation pathways are altered by stabilization of CTNNB1 in the yolk sac epithelium}

To identify functional changes in the yolk sac epithelium, RNAseq was performed on RNA isolated from

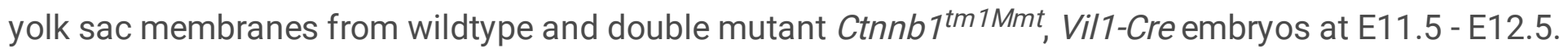
Hierarchical clustering of differentially expressed genes separated the mutant from wildtype samples with two mutant samples appearing to be outliers (Fig. 2A), which was confirmed by PCA (Fig. 2B). After the outliers were removed, PCA and hierarchical clustering showed clustering by genotype (Fig. 2C, D).

The functional categories of the differentially expressed genes were analyzed using Ingenuity Pathway Analysis (IPA). The differentially expressed genes were enriched for pathways connected to inflammatory responses (Fig. 3A). The top affected pathways were Granulocyte Adhesion and Diapedesis and Agranulocyte Adhesion and Diapedesis, both of which are associated with inflammation and macrophage activity. Inflammatory response was the top functional category $\left(p=1.84 \times 10^{-30}\right.$, Fig. 3B), and the top predicted upstream regulator of the differentially regulated genes was tumor necrosis factor $\left(\right.$ TNFA) $\left(p=2.27 \times 10^{-41}\right.$, Fig. $\left.3 C\right)$.

\section{Discussion}

We show that induction of ectopic WNT signaling by CTNNB1 stabilization in the yolk sac epithelium leads to embryonic lethality, likely due to compromised hematopoiesis. The disruption of yolk sac function would be fatal to the embryo, since it provides an essential function retaining and releasing HSCs that are required for early embryonic survival. Taken together, our data suggests that embryo lethality caused by accumulation of hematopoietic precursors in the yolk sac epithelium due to absence of a functional circulation [11].

The first developmental hematopoietic activity occurs in the extra-embryonic yolk sac, which is crucial to the development of the early embryo and ensures embryonic survival by acting as a temporary substitute for a hematopoietic system before a permanent system can be established by hematopoietic stem cells [10]. The yolk sac derived hematopoiesis takes place in two distinct waves. The first wave begins around E7.25 and includes primitive macrophage progenitors that colonize the brain through the vasculature [10, 12], while the second wave of erythromyeloid progenitors (EMPs) arises around E8.25 and colonizes the fetal liver before the arrival of hematopoietic stem cells (HSCs) [10]. Although conflicting, several studies have reported HSCs to appear at E10.5-11.5 in multiple locations including the yolk sac [13] which is 
consistent with our bioinformatic analysis showing activation of regulatory proinflammatory cytokines, whose production and secretion are dependent on NFKB activation in HSCs. Additionally, it has been demonstrated that HSCs rapidly respond to IFNG, which acts downstream by activating STAT3 $[14,15]$. Both molecules are predicted to be activated by IPA analysis. All these factors contribute to HSCs specification, emergence, maturation and expansion. The last two phases will take place into the developing fetal liver and determine the maturation of myeloid and lymphoid cells all necessary for embryonic survival $[16,17]$. Our IPA analysis has suggested increased migration and homing of cells among the altered functions.

Several studies have investigated the dependency on WNT signaling during mouse embryonic development. Ctnnb $1^{\text {tm } 1 \mathrm{Mmt}}$ deletion in endothelial cells causes abnormal vascularization in mouse embryos, resulting in resorption at E12.5 [18]. Homozygous Fzd5 knockout mice, in which WNT signaling is disrupted, are embryonically lethal at E10.75 due to defects of yolk sac vasculogenesis [19]. Additionally, selective stabilization of CTNNB1 in endothelial cells caused embryonic death, which was also observed around E12.5 along with aberrant vascular development of the embryo and the yolk sac membrane [20].

\section{Conclusions}

Normal levels of WNT signaling is required during yolk sac-mediated hematopoiesis and may contribute to abnormal pregnancy outcomes with aberrant levels. Our data also shows the limitation of use the Vil1Cre transgenic line in for developmental inactivation of specific genes.

\section{Methods}

\section{Mice and crosses}

All mice were purchased from The Jackson Laboratory (Bar Harbor, ME) are maintained in house on a C57BL/6J genetic background. B6.129X1-Ctnnb $1^{\text {tm 1Mmt }}$ mice, with flox sites flanking exon 3, were crossed with B6N.Cg-Tg(Vil1-cre)20Syr/J mice that express CRE recombinase under an intestinal epithelial promoter. Control mice were littermates lacking the cre transgene and the experimental unit was individual mice and both sexes were used. Samples sizes were based on prior experience with mutant moue analysis and no confounding criteria were used. No blinding of samples was used since there was no subjective analyses. The beta-galactosidase reporter B6.129S4-Gt(ROSA)26Sortm $1 \mathrm{Sor} / \mathrm{J}$ was crossed with Vil1-Cre to determine the location of CRE expression. Embryos were collected at E10.5-E13.5 based

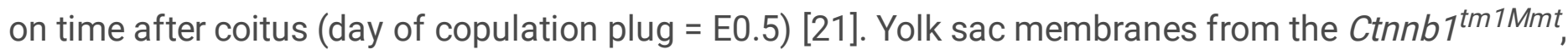
Vil1-Cre cross were collected for both histological and RNAseq analyses. Embryos and extras-embryonic tissues were stained using an established beta-galactosidase staining protocol [22]. Mice were euthanized by $\mathrm{CO}_{2}$ asphyxiation using a protocol approved by the Texas A\&M Institutional Animal Care and Use Committee. 


\section{Genotypes}

DNA was isolated from tissue samples using the Promega Maxwell DNA Extraction/Purification Kit on a Promega Maxwell 16 instrument. Genotypes were determined by PCR using the following primers: Ctnnb1-WT forward: 5' - GGTAGGTGAAGCTCAGCGCAGAGC- 3'; Ctnnb1-WT reverse: 5' ACGTGTGGCAAGTTCCGCGTCATCC- 3'; Ctnnb1-Floxed forward: 5' -TTTCCCAGTCCTTCACGCAAGAGC- 3'; Ctnnb1-Floxed reverse: 5' -ATCGCCTTCTATCGCCTTCTTGACG- 3'; Cre Forward: 5' GCGTGGCAGTAAAAACTTC- 3'; Cre Reverse: 5' -GTGAAACAGCATTGCTGTCACTT- 3'; Wt forward: 5' CTAGGCCACAGAATTGAAAGATCT- 3'; Wt reverse: 5' -GTAGGTGGAAATTCTAGCATCATCC- 3'.

\section{RNA Isolation, sequencing, and analysis}

RNA sequencing was performed by the Molecular Genomics Core of the Texas A\&M Institute for Genome Sciences and Society to assess differential gene expression. RNA was isolated using the Trizol Reagent (Invitrogen), following the manufacturer's protocol. RNA samples were quantified via RNA Qubit assay (ThermoFisher), and the quality of the RNA was checked using an Agilent 4200 TapeStation. All samples met appropriate concentrations and quality standards for mRNA library preparation. mRNA sequencing libraries were prepared using the Illumina TruSeq mRNA library preparation kit (Illumina) following the manufacturer's protocol. Libraries were pool in equimolar concentrations for sequencing on an Illumina NextSeq 500 75nt single end sequencing run producing approximately 50 million reads for each sample.

A total of approximately 548 million 75 base-pair single-end reads from 10 samples were checked to trim any adapter sequences and low-quality bases using Trimmomatic [23] resulting in approximately 477 million filtered reads ( $87 \%$ ) out of which a total of 366 million filtered reads (approximately $76.6 \%$ ) uniquely mapped to the reference sequences of Mus Musculus (mm10 ENSEMBL release 99) genome assembly. Read mapping was performed using STAR [24]. Transcript-wise counts were generated using feature Counts tool from the SUBREAD package [25]. Differential gene expression tests were then performed used DESeq2 (Love, Huber et al. 2014) following recommended guidelines by the authors. Gene expression profiles of mutant samples and controls were compared to select differentially expressed transcripts with an absolute fold-change $\geq 2$ and corrected $p$-values $\leq 0.01$. Data was analyzed using the Ingenuity Pathway Analysis software (QIAGEN). Plots were generated using R.

\section{Declarations}

Ethics approval: All animal studies were approved by the Texas A\&M Institutional Animal Care and Use Committee.

Consent for publication: Not applicable.

Availability of data and materials: All mouse lines are available from public repositories. All data is freely available from the authors.

Competing interests: The authors have no conflicts of interest to declare. 
Funding: This work was supported by National Institutes of Health grants RM1 HG008529, R25 CA172010, and P30 ES029067 to DWT and F32 CA168301 to RML. The funders were not involved in the design of the study, collection, analysis, and interpretation of data, or the writing of the manuscript.

Authors' contributions: RML and DWT conceived the study; RML, MB, and DC performed the experiments; MB, DC, and DWT prepared the manuscript; all authors edited the manuscript.

Acknowledgments: We thank members of the Threadgill Lab for assistance with animal work, and informatic discussions.

\section{References}

1. Metcalfe C, Bienz M: Inhibition of GSK3 by Wnt signalling-two contrasting models. J Cell Sci 2011, 124(Pt 21):3537-3544.

2. Haegel $H$, Larue L, Ohsugi M, Fedorov L, Herrenknecht $K$, Kemler R: Lack of beta-catenin affects mouse development at gastrulation. Development 1995, 121(11):3529-3537.

3. Forbes SA, Bindal N, Bamford S, Cole C, Kok CY, Beare D, Jia M, Shepherd R, Leung K, Menzies A et al: COSMIC: mining complete cancer genomes in the Catalogue of Somatic Mutations in Cancer. Nucleic Acids Res 2011, 39(Database issue):D945-950.

4. Basu S, Haase G, Ben-Ze'ev A: Wnt signaling in cancer stem cells and colon cancer metastasis. F1000Res 2016, 5.

5. Hay-Koren A, Caspi M, Zilberberg A, Rosin-Arbesfeld R: The EDD E3 ubiquitin ligase ubiquitinates and up-regulates beta-catenin. Mol Biol Cell 2011, 22(3):399-411.

6. Xia J, Urabe K, Moroi Y, Koga T, Duan H, Li Y, Furue M: beta-Catenin mutation and its nuclear localization are confirmed to be frequent causes of Wnt signaling pathway activation in pilomatricomas. J Dermatol Sci 2006, 41(1):67-75.

7. Hamada S, Futamura N, Ikuta K, Urakawa H, Kozawa E, Ishiguro N, Nishida Y: CTNNB1 S45F mutation predicts poor efficacy of meloxicam treatment for desmoid tumors: a pilot study. PLoS One 2014, 9(5):e96391.

8. MacDonald BT, Tamai K, He X: Wnt/beta-catenin signaling: components, mechanisms, and diseases. Dev Cell 2009, 17(1):9-26.

9. Tran HT, Sekkali B, Van Imschoot G, Janssens S, Vleminckx K: Wnt/beta-catenin signaling is involved in the induction and maintenance of primitive hematopoiesis in the vertebrate embryo. Proc Natl Acad Sci U S A 2010, 107(37):16160-16165.

10. Frame JM, Fegan KH, Conway SJ, McGrath KE, Palis J: Definitive Hematopoiesis in the Yolk Sac Emerges from Wnt-Responsive Hemogenic Endothelium Independently of Circulation and Arterial Identity. Stem Cells 2016, 34(2):431-444.

11. Rhodes KE, Gekas C, Wang Y, Lux CT, Francis CS, Chan DN, Conway S, Orkin SH, Yoder MC, Mikkola HK: The emergence of hematopoietic stem cells is initiated in the placental vasculature in the 
absence of circulation. Cell Stem Cell 2008, 2(3):252-263.

12. Stremmel C, Schuchert R, Wagner F, Thaler R, Weinberger T, Pick R, Mass E, Ishikawa-Ankerhold HC, Margraf A, Hutter S et al: Author Correction: Yolk sac macrophage progenitors traffic to the embryo during defined stages of development. Nat Commun 2018, 9(1):3699.

13. Huang $\mathrm{H}$, Auerbach $\mathrm{R}$ : Identification and characterization of hematopoietic stem cells from the yolk sac of the early mouse embryo. Proc Natl Acad Sci U S A 1993, 90(21):10110-10114.

14. Li Y, Esain V, Teng L, Xu J, Kwan W, Frost IM, Yzaguirre AD, Cai X, Cortes M, Maijenburg MW et al: Inflammatory signaling regulates embryonic hematopoietic stem and progenitor cell production. Genes Dev 2014, 28(23):2597-2612.

15. Sawamiphak S, Kontarakis Z, Stainier DY: Interferon gamma signaling positively regulates hematopoietic stem cell emergence. Dev Cell 2014, 31(5):640-653.

16. Houssaint E: Differentiation of the mouse hepatic primordium. II. Extrinsic origin of the haemopoietic cell line. Cell Differ 1981, 10(5):243-252.

17. Zovein AC, Turlo KA, Ponec RM, Lynch MR, Chen KC, Hofmann JJ, Cox TC, Gasson JC, Iruela-Arispe $\mathrm{ML}$ : Vascular remodeling of the vitelline artery initiates extravascular emergence of hematopoietic clusters. Blood 2010, 116(18):3435-3444.

18. Cattelino A, Liebner S, Gallini R, Zanetti A, Balconi G, Corsi A, Bianco P, Wolburg H, Moore R, Oreda B et al: The conditional inactivation of the beta-catenin gene in endothelial cells causes a defective vascular pattern and increased vascular fragility. J Cell Biol 2003, 162(6):1111-1122.

19. Ishikawa T, Tamai Y, Zorn AM, Yoshida H, Seldin MF, Nishikawa S, Taketo MM: Mouse Wnt receptor gene Fzd5 is essential for yolk sac and placental angiogenesis. Development 2001, 128(1):25-33.

20. Corada M, Nyqvist D, Orsenigo F, Caprini A, Giampietro C, Taketo MM, Iruela-Arispe ML, Adams RH, Dejana E: The Wnt/beta-catenin pathway modulates vascular remodeling and specification by upregulating DII4/Notch signaling. Dev Cell 2010, 18(6):938-949.

21. Davenport TG, Jerome-Majewska LA, Papaioannou VE: Mammary gland, limb and yolk sac defects in mice lacking Tbx3, the gene mutated in human ulnar mammary syndrome. Development 2003, 130(10):2263-2273.

22. Burn SF: Detection of beta-galactosidase activity: X-gal staining. Methods Mol Biol 2012, 886:241250.

23. Bolger AM, Lohse M, Usadel B: Trimmomatic: a flexible trimmer for Illumina sequence data. Bioinformatics 2014, 30(15):2114-2120.

24. Dobin A, Davis CA, Schlesinger F, Drenkow J, Zaleski C, Jha S, Batut P, Chaisson M, Gingeras TR: STAR: ultrafast universal RNA-seq aligner. Bioinformatics 2013, 29(1):15-21.

25. Liao Y, Smyth GK, Shi W: featureCounts: an efficient general purpose program for assigning sequence reads to genomic features. Bioinformatics 2014, 30(7):923-930.

\section{Figures}


A
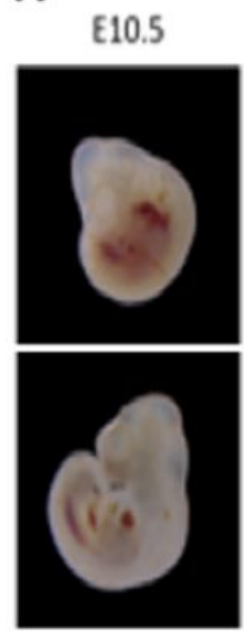

E12.5
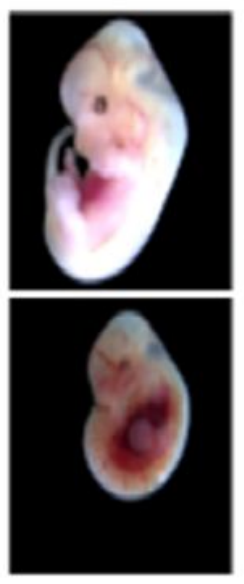

E135
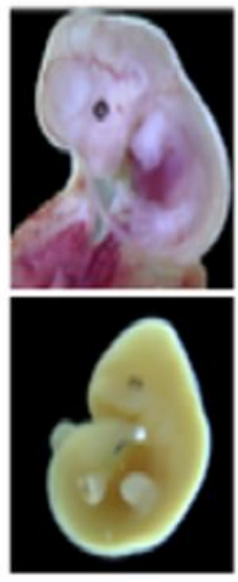

B

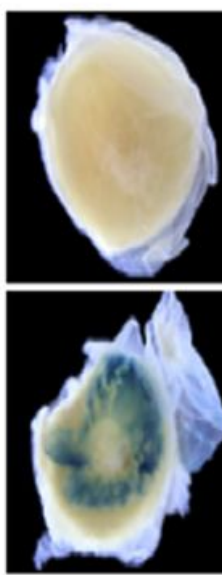

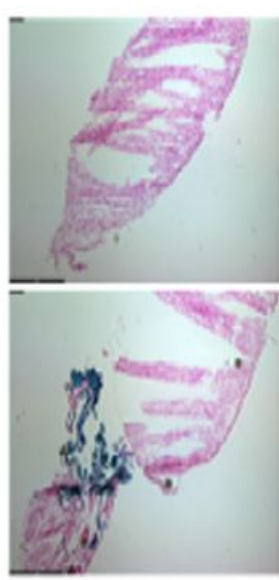

Gt(ROSA)26Sor ${ }^{r m 150 x}$ Vil1-Cre

\section{Figure 1}

Developmental analysis. A Development of control and mutant Ctnnb1tm1Mmt, Vil1-Cre embryos. B Betagalactosidase staining of placental tissue from showing Vil1-Cre expression. 

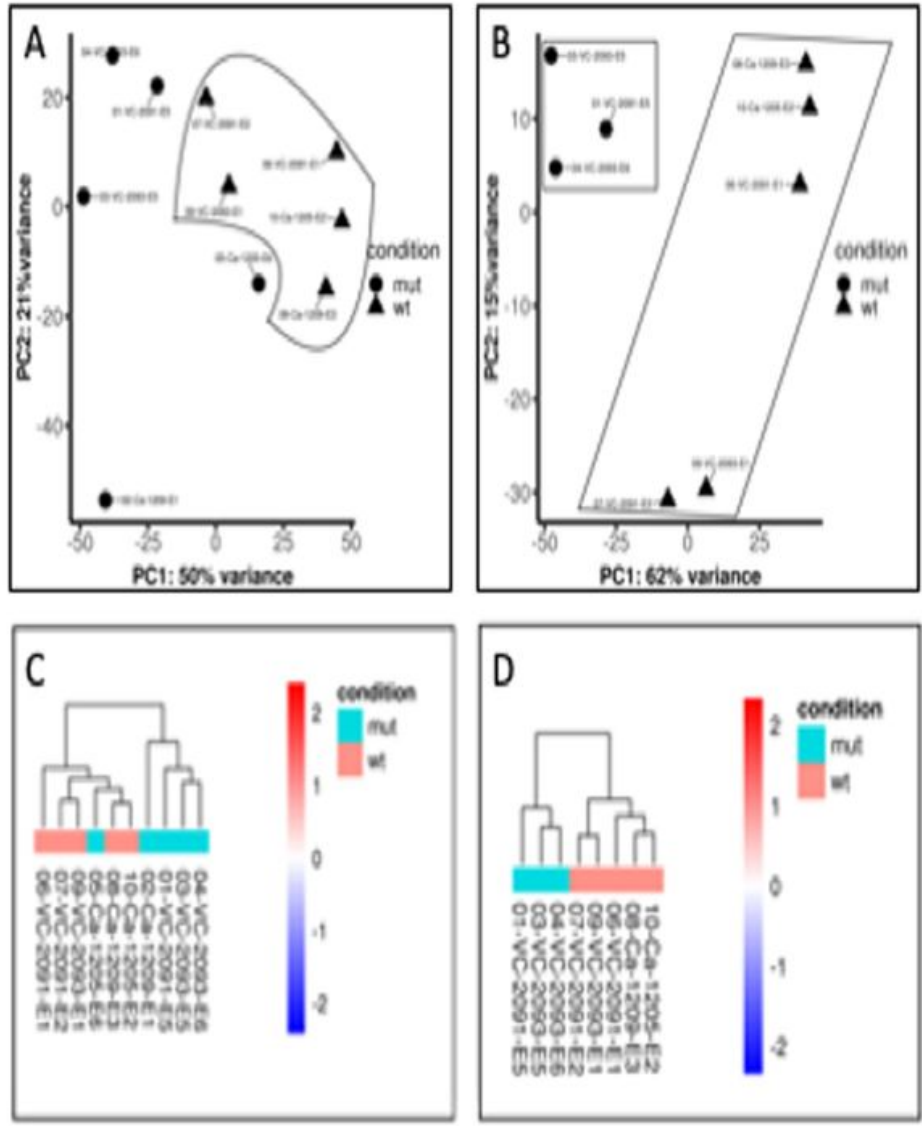

Figure 2

Analysis of RNAseq data. A, Initial PCA plot of all collected samples. B, PCA plot after removal of outliers. C, Initial cluster plot of all collected samples. D, Cluster plot after removal of outliers. 


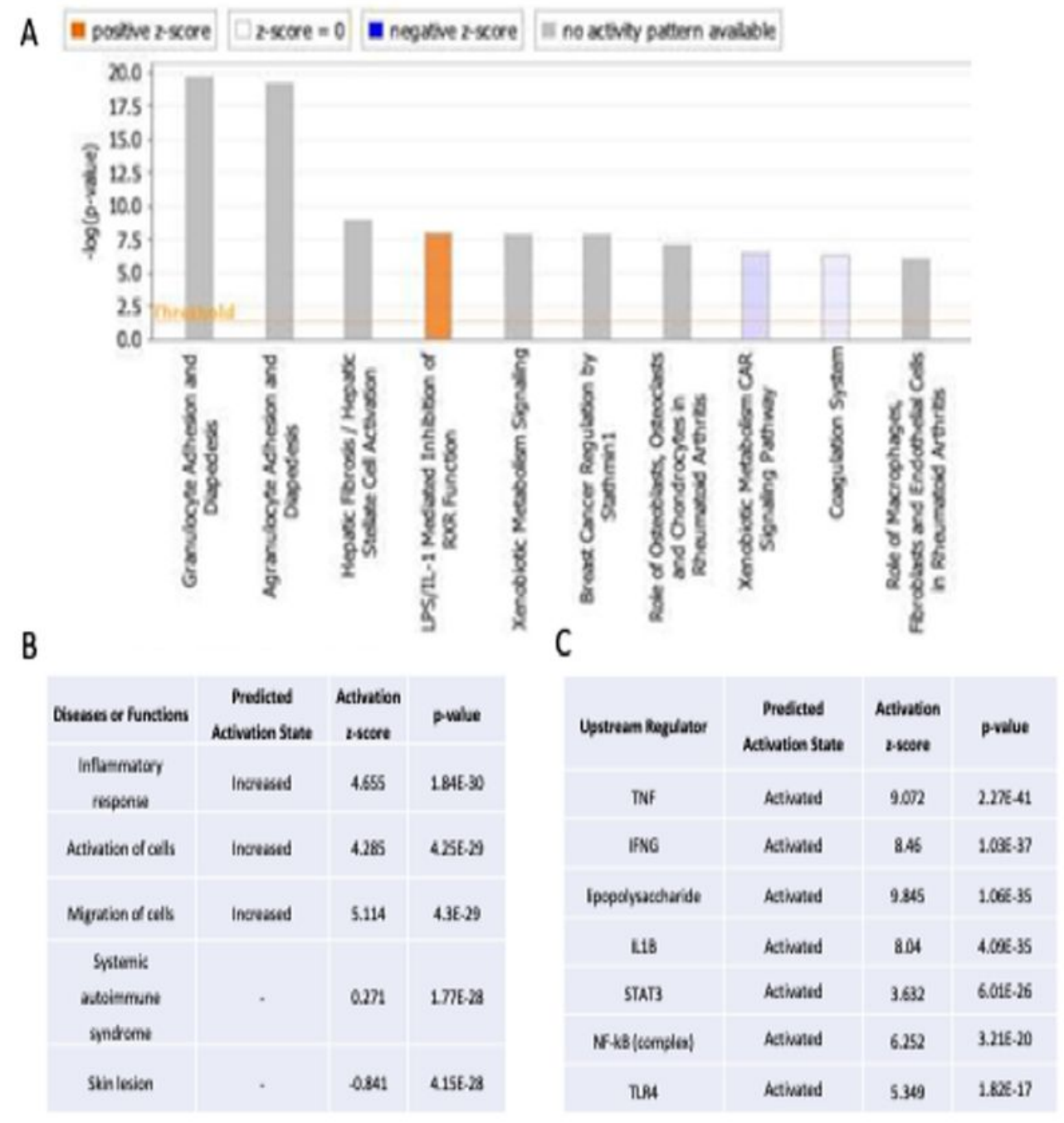

\section{Figure 3}

Analysis of RNAseq data using IPA. A Top 10 enriched diseases and cellular functions. B Top 5 enriched diseases and functions. C Top 7 predicted upstream regulators. 